made with the results of investigations of the angular correlation of positron annihilation radiation (Berko, Proc. 5th Int. Conf. Positron Annihtlation, Japan 1979, 8A-II-3).

Considerable progress in the field of charge and momentur density studies is expected once Synchrotron radiation and instrumentation adapted to its properties is in use. A 5-circle diffractometer installed at HASYLAB (Hamburg) will be described. The first Compton profile measurements performed at LURE (Paris) on Be metal (Loupias, Petiau, Issolah \& Schneider, M., phys. stat. sol. b102 (1980) 79) will be compared with recent $\gamma$-ray and $x$-ray measurements. In the near future it will still be difficult to extend the photon Compton scattering technique to thin film studies. Here high energy electron compton scattering may become a useful tool, provided the problem of radiation damage can be solved. Another possibility is to explore the interaction of fast ions with thin foils which leads to radiative electron capture (Spindler, Betz \& Bell, J. Phys, B 10 (1977), 1561). Again radiation damage limits the range of applications and further cross-section studies are necessary.

06.X-11 ANALYSIS OF THE ELECTRONIC STRUCTURE BASED ON DIFFRACTION AND COMPTON SCATTERING MEASUREMENTS. EXAMPLES: Be AND $\mathrm{Li}_{3} \mathrm{~N}$.

BY N.K. Hansen, Hahn-Meitner-Institut für Kernforschung, Berlin, FRG

We wish to discuss how combined information from diffraction and compton scattering experiments may be used to obtain a better understanding of chemical bonding in solids. Two examples will be presented: Be, a metal, and $\mathrm{Li}_{3} \mathrm{~N}$, a fast ionic conductor.

For Be the most recent $X$-ray structure factor measurements (Larsen \& Hansen, to be published) are in disagreement with P.J. Brown's previous measurements (Phil.Mag. (1972)26,1377). The earlier data were interpreted as indicating a predominant $\mathrm{SP}_{z}$ hybridization, whereas the new data show only a very small deviation from superimposed free atom densities. On the other hand, the Compton profiles for Be can certainly not be described by free atoms. In order to obtain reasonable agreement with the Compton profiles, we must start from another model (e.g. a free electron gas) or go through a procedure which includes explicitly corrections for solid state effects on the atomic model. We have constructed a simple renormalised free atom model for the 1electron wave functions which does give rise to some of the right trends both for the structure factors as well as for the compton profiles, but which certainly does not exhaust the available information.

Ii $3^{\mathrm{N}}$ will be used to demonstrate how effects of short and long range interactions can be separated in the Fourier transform of the compton profile, and how sensitive it is to the nonbonding interactions in this crystal. The analysis supports the conclusions based on $N Q R$ and $X$-ray diffraction results: The bonding in $\mathrm{Li}_{3} \mathrm{~N}$ is basicly ionic with evidence of considerable distortion of the $\mathrm{N}^{3-}$ ion due to wave function overlap with neighbouring ions. It was important for the understanding of the electronic structure that various theoretical models at different levels of sophistication were available, including a recent self-consistent pseudopotential calculation (Kerker, MPI, Stuttgart, to be pubIished).

\section{X-12 X-RAY DIFFRACTION STUDIES OF SURFACES AND} INTERFACES. By P. Eisenberger, Bell Laboratories, Murray $\mathrm{Hi} 11$, NJ 07974, U.S.A.

Using glancing angle Bragg diffraction including the use of total external reflection, one has enough sensitivity with modem high powered $\mathrm{X}$-ray sources to study monolayer structures or buried thin interfaces. Results on reconstructed $\mathrm{Ge}(100)$ and on the Al/GaAs interface will be discussed. 